\title{
BILATERAL NASOLABIAL CYST: A RARE CAUSE OF NASAL OBSTRUCTION
}

Samir Choudhary ${ }^{1}$

\section{HOW TO CITE THIS ARTICLE:}

Samir Choudhary. "Bilateral Nasolabial Cyst: A Rare Cause of Nasal Obstruction". Journal of Evolution of Medical and Dental Sciences 2015; Vol. 4, Issue 86, October 26; Page: 15105-15109, DOI: 10.14260/jemds/2015/2145

\begin{abstract}
Nasolabial cyst is rare, nonodontogenic, soft tissue, and developmental cyst. It is located inferior to the nasal ala. It is frequently asymptomatic, we report a rare case of bilateral nasolabial cysts causing nasal obstruction. This case report provides review of current knowledge adout pathogenesis, symptoms, imaging modalities, histopathology, treatment options and prognosis of nasolabial cyst. A 30 years old woman presented with swelling below the nose around the upper lip with facial asymmetry and nasal obstruction since one year. On Physical examinations, there was elevation of nasal bilateral nasal alae and effacement of nasolabial folds. CT scan paranasal sinuses revealed bilateral nasolabial cysts occupying anterior and inferior part of nasal cavity, the cysts were ressected completely via sublabial approach with uneventful postoperative period.
\end{abstract}

KEYWORDS: Nasolabial fold, Nonodontogenic cysts, Nasal obstruction.

INTRODUCTION: Nasolabial cyst is a rare, nonodontogenic maxillofacial soft tissue, developmental cyst that accounts for $0.7 \%$ of all jaw cysts.1,2 and $2.5 \%$ of nonodontogenic cysts. It was first described by Zukerkandl in $1882 .{ }^{3}$ the term Nasolabial cyst itself was coined by Rao. ${ }^{4}$ It is an extraosseous cyst located in the area of nasolabial fold.

Nnasolabial cyst is usually unilateral (90\%), more commonly located on left side. It affects mainly black races and frequent in females in proportion of 4:1 when unilateral and 5.5: 1 when bilateral. The age group most affected include fourth and fifth decade and exceedingly uncommon in patients younger than 10 years. If not infected it is painless swelling, typically appear as a swelling at canine fossa, gingivobuccal sulcus elevating ala of nose. Depending on how much nasal cavity is affected, it may lead to nasal obstruction. It may rarely affect lacrimal drainage causing dacryocystitis and symptomatic epiphora.

Despite the uncommon occurrence it is important to know the characteristics of the nasolabial cyst. Here we report a rare case of bilateral nasolabial cyst, its clinical presentation, radiological and histopathological features and surgical treatment options.

CASE REPORT: A 30 years old female presented in August 2015 with complaints of swelling around the upper lip on both sides with facial asymmetry since one year. It was gradually progressive and painless. She developed nasal obstruction since last three months. On examination, there was swelling at bilateral nasolabial regions obliterating the nasolabial folds. Both nasal alae were elevated blocking left nasal vestibule completely and right one partially. Intraorally, there was obliteration of superior gingivolabial sulcus bilaterally contiguous to maxillary incisors and canines. On palpation the lesion on both sides was cystic, fluctuant and non-tender. A working diagnosis of bilateral nasolabial cysts was made. To differentiate between odontogenic and other non-odontogenic lesion, CT scan Paranasal sinuses with IV contrast was done which revealed homogenous oval cystic lesions anterior to the nasal apertures, of size $3 \times 2 \mathrm{~cm}$ on left side and $2 \times 2 \mathrm{~cm}$ on right side.

The masses had pushed anterior maxillary wall of both sides posteriorly with scalloping, occupying inferior and anterior portion of nasal cavities. Patient was hospitalized and surgical 
excision of both lesion was done by intraoral approach under general anaesthesia. Sublabial incision was given over cystic lesions from lateral incisior on right side to canine on left side.

Two lesions were found to be separated by a bony septum and there was scalloping of anterior wall of both maxillae. Both cysts were dissected out in toto. Left sided cyst was of size $3 \times 2 \mathrm{~cm}$ and right nasolabial cyst was of $2 \times 2 \mathrm{~cm}$. Wound was sutured in layers with absorbable sutures. Postoperative healing period was uneventful.

Histopathological study was identical in both lesions. The cystic wall was constituted by loose connective tissue covered by cuboidal basal layer and a columnar luminal layer with interspersed scattered mucinous goblet cells confirming the diagnosis of nasolabial cyst.

DISCUSSION: The nasolabial cyst is an embryonic non-odontogenic cyst. Its pathogenesis is still uncertain. There are two theories regarding the pathogenesis of nasolabial cyst. According to first theory, the cyst arises as a fissural cyst which develops from embryonic nasal epithelium entrapped between merging maxillary processes and the medial lateral nasal processes. ${ }^{5}$ the other affirms that the lesion is a developmental cyst arising from the remnant of the embryonic nasolacrimal duct due to histopathological similarity to the later. ${ }^{6}$ This theory is the most accepted one.

Being asymptomatic, nasolabial cyst is under-diagnosed. Patients seek medical advice when the cyst becomes infected causing pain, or cause cosmetic defect. The lesion may rarely leads to nasal obstruction, chronic dacryocystitis due obstruction of lacrimal drainage. Infected cyst sometimes rupture spontaneously to the oral cavity of to the nasal vestibule. The case presented here is uncommon for being bilateral cyst and causing nasal obstruction. It has been estimated that approximately $10 \%$ of nasolabial cysts are bilateral. ${ }^{7}$

The nasolabial cysts are underdetactable on plain radiography except in the event of maxillary erosion. Conversely, Seward. ${ }^{8}$ described two radiological aspects: increased radiolucency adjacent to the apical region of the incisors and deformity of the radiopaque line corresponding to the inferior border of pyriform aperture. On CT scan, the cyst appears homogenous cystic rounded lesion anterior to the pyriform aperture. Large lesions may be associated with bone remodelling of the underlying maxilla. On MRI, they appear rounded hypointense soft tissue on T1 (Slightly hyperintense to the CSF) and bright hyperintense on T2 (Isointense to the CSF) on the anterior part of floor of nasal cavity In the case reported here, lesions on both sides were showing same CT characters with scalloping of left maxillary anterior wall. Histopathologically lining of the cyst is pseudostratified columnar epithelium with numerous goblet cells. It can also be lined by bilayered epithelium with a cuboidal basal layer and columnar luminal layer, or lining may be multilayered, cuboidal to columnar. ${ }^{9}$

Due to similar signs and symptoms, nasolabial cyst may be misdiagnosed as radicular cyst, periapical abscess, odontogenic cyst such as dentigerous cyst, nasopalatine duct cyst, benign soft tissue tumours of minor salivary gland, dermoid and epidermoid cyst. Radicular cyst arises from maxillary anterior teeth and extends to adjacent soft tissues. Vitality sign and presence of radiolucency in relation to apices of involved teeth can rule out the diagnosis. Periapical abscess from anterior maxillary tooth is a inflammatory lesion. Presence of nonviable tooth differentiates it from nasolabial cyst. Nasopalatine duct cyst is intraosseus cyst found in the anterior midline of palate and/ or the midline labial aspect of alveolar ridge.

Whereas nasolabial cyst is the extraosseous cyst, found in soft tissue. Dentigerous cyst encloses the crown of unerrupted tooth by expansion of its follicle and attached to the neck. On 
radiography presence of radiolucency and intraosseous location are the differentiating points from nasoladial cyst. Benign soft tissue tumours and minor salivary gland tumours differentiated from nasolabial cyst by their solid consistency and lack of enhancement. Also fluid level is not seen in those tumours. Dermoid and epidermoid cysts are seen in the midline of the nose or laterally below nasal ala. They are usually diagnosed in the childhood whereas nasolabial cyst is more common in adults.

Treatment of choice for nasolabial cyst is surgical excision via sublabial approach. During excision of large cysts extending to the nasal floor, dissection should be done carefully to avoid perforation. In the case presented here, the cyst was bilateral. So the sublabial incision was between the level of lateral incisor on right side to canine on left side, of approximate size of $5 \mathrm{~cm}$. If complete resection is achieved, recurrence is extremely rare. Alternativey, transnasal endoscopic marsupulization of cyst can be considered for treatment.10 other treatment modalities include, injection of sclerosing agent, aspiration and incision and drainage. However all of these especially the later two carry the risk of recurrence and are rarely used. With adequate treatment prognostic and esthetic outcome of patients of nasolabial cyst is excellent.

So in the presented case, clinical, radiological and histopathological features were suggestive of bilateral nasolabial cyst, which is a rare finding. Both the cysts were successfully ressected completely by sublabial approach and with no evidence of complications.

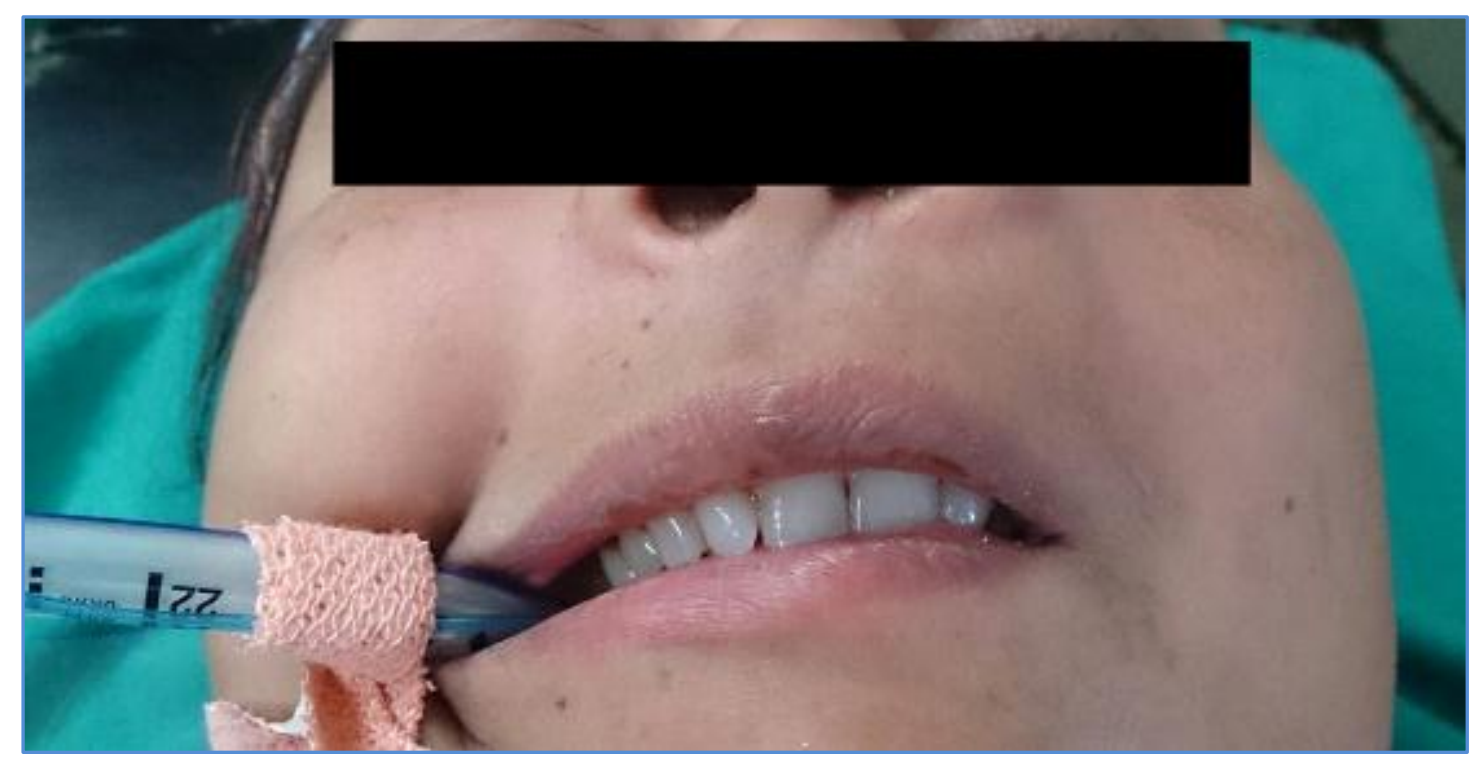

Fig. 1: Clinical Picture showing bilateral extra oral swelling on Both sides with Elevation of both ala of nose more on left side 


\section{CASE REPORT}

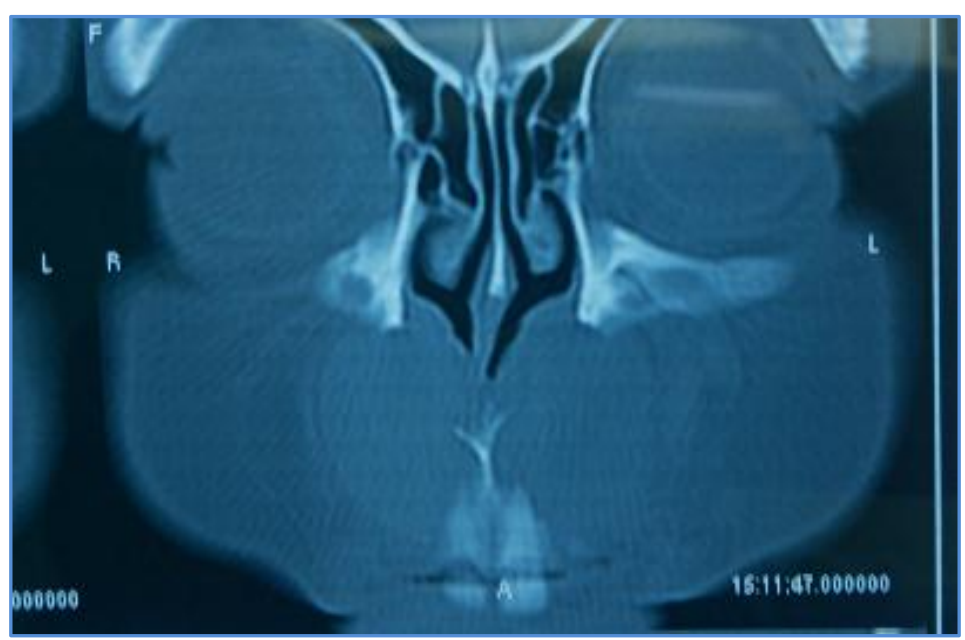

Fig. 2: Coronal CT scan PNS Plain showing bilateral nasolabial cysts

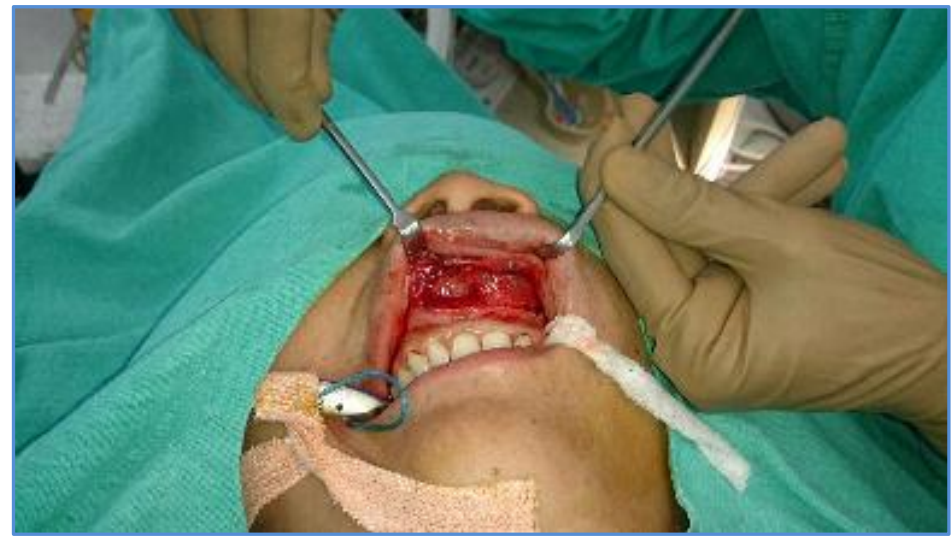

Fig. 3: Exposure of bilateral cyst capsule by sublabial incision

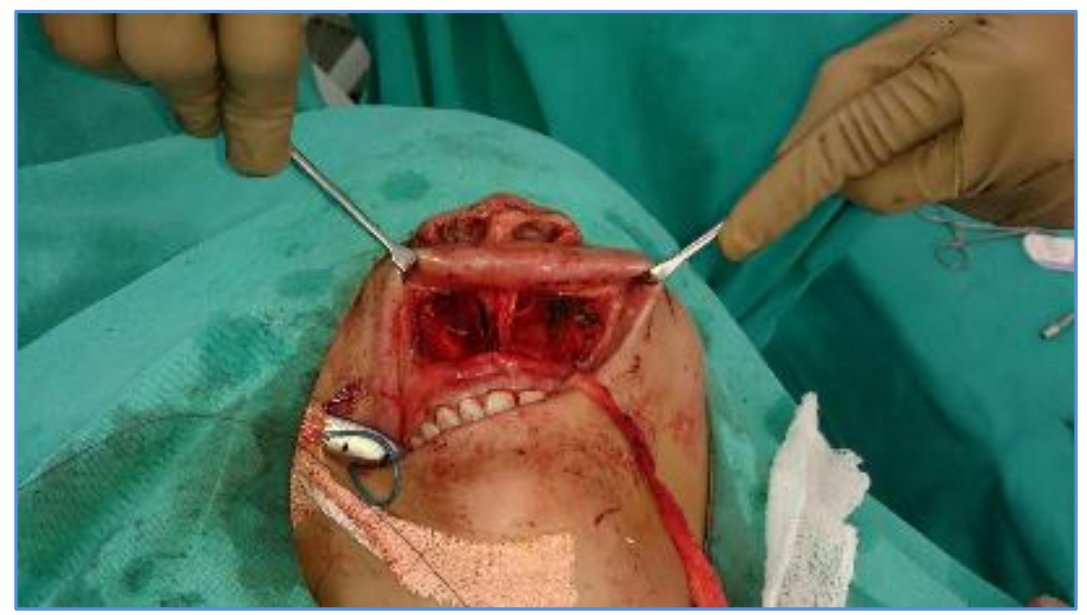

Fig. 4: The operative field after resection of bilateral Nasolabial cysts, showing scalloping of bone 


\section{REFERENCES:}

1. Chinellato LE, Damante JH. Contribution of radigraphs to the diagnosis of nasoalveolar cyst. Oral Surg Oral Med Oral Pathol. 1984; 58:729-735.

2. Wesley RK, Scannell T, Nathan LE. Nasolabial cyst: presentation of a case with a review of the literature. J Oral Maxillofac Surg. 1984; 42:188-192.

3. Zuckerkandl E. Normale und pathologische Anatomie der Nasenhohle. Vienna: W. Braunmuller. 1882.

4. Rao RV. Nasolabial cyst. J Laryngol Otol. 1955; 69:353-4.

5. Klestadt WD. Nasal cysts and the facial cleft cyst theory. Ann Otol Rhinol Laryngol. 1953; 62:8492.

6. Van Bruggemann A. Cysts as a result of developmental problems in the nasal vestibule. Arch Oto Rhino Laryn. 1920; 33:103-19.

7. Roed-Peterson B. Nasolabial cysts: a presentation of five patients with a review of literature. Br. J. Oral Surg. 1969; 7:84-95.

8. Seward GR. Nasolabial cysts and their radiology. Dent Pract. 1962; 12:154-61.

9. Yanelba Toribio, Micheal HA, Roehrl. The nasolabial cyst: a nonodontogenic oral cyst related to nasolacrimal duct epithelium.2011; 135(11):1499-1503.

10. Su CY, Chien CY, Hwang CF. A new transnasal approach to endoscopic marsupialisation of the nasolabial cyst Laryngoscope. 1999; 109: 1116-1118.

\section{AUTHORS:}

1. Samir Choudhary

PARTICULARS OF CONTRIBUTORS:

1. Consultant ENT and Head Neck Surgeon, Choudhary ENT Clinic, Ramdaspeth, Nagpur.

FINANCIAL OR OTHER COMPETING INTERESTS: None

\section{NAME ADDRESS EMAIL ID OF THE} CORRESPONDING AUTHOR:

Dr. Samir Choudhary,

No. 12, Saraswati Vihar, Trimurti Nagar, Nagpur-440022.

E-mail: samirchoudhary45@yahoo.com

Date of Submission: 01/10/2015.

Date of Peer Review: 03/10/2015.

Date of Acceptance: 15/10/2015.

Date of Publishing: 26/10/2015. 